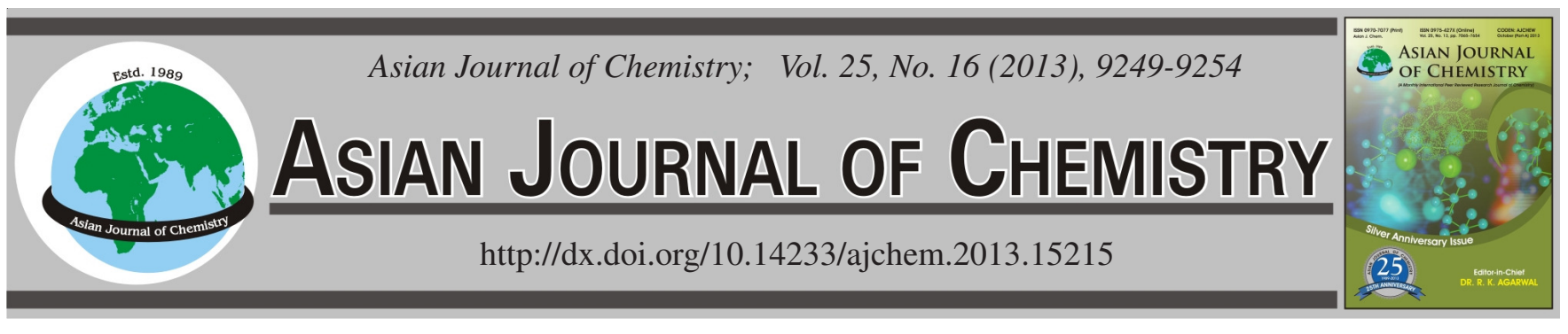

\title{
2,2-Diphenyl-1-Picrylhydrazyl Free Radical Scavenging Assay and Bacterial Toxicity of Protein Capped Silver Nanoparticles for Antioxidant and Antibacterial Applications
}

\author{
G. Rajasekhar Reddy, Antony Bertie Morais and N. Nagendra Gandhi*
}

Department of Chemical Engineering, Alagappa Chettiar College of Technology, Anna University, Chennai-600 025, India

*Corresponding author: E-mail: n_nagendra2002@yahoo.com; grajasekharau@gmail.com

\begin{abstract}
Current study deals with the synthesis, in vitro antioxidant and bacterial toxicity of silver nanoparticles using aqueous extract of Senna siamea plant seed. The reaction mixture turned to brownish colour after $2 \mathrm{~h}$ of incubation and was confirmed by surface Plasmon spectra using UV-visible spectrophotometer around $440 \mathrm{~nm}$ characteristic of silver nanoparticles. The stability of silver nanoparticles is due to the high negative values of zeta potential and capping of phytoconstituents which is present in the aqueous extract of S. siamea seed which is evident from Fourier transform infrared spectroscopy studies. Dynamic light scattering (DLS) studies revealed that the diameter of stable silver nanoparticles was approximately $70 \mathrm{~nm}$ in size and $-22.9 \mathrm{mV}$ zeta potential value with high stability. High resolution transmission electron microscopy analysis showed that the silver nanoparticles were poly dispersed with spherical shape. X-Ray diffraction studies revealed that most of the nanoparticles were face centered cubic in shape. Synthesized silver nanoparticles showed effective antioxidant activity against to 2,2-diphenyl-1-picrylhydrazyl (DPPH) free radical scavenging assay. Phytosynthesized silver nanoparticles showed potent antibacterial activity against two gram positive (Staphylococcus aureus and Bacillus subtilis) and three gram negative (Escherichia coli, Pseudomonas aeruginosa and Klebsiella pneumonia) human pathogenic bacteria which leads towards the clinical use as antibacterial agent.
\end{abstract}

Key Words: Silver nanoparticles, Antioxidant, Zeta potential, Antibacterial activity.

\section{INTRODUCTION}

Nanoparticles exhibit new and improved properties based on size, distribution and morphology than larger particles of the bulk materials from which the nanoparticles are made ${ }^{1}$. The surface to volume ratio of nanoparticles is inversely proportional to their size. The biological effectiveness of nanoparticles can increase proportionately with an increase in the specific surface area due to the increase in their surface energy and catalytic reactivity ${ }^{2}$. Studies have shown that the size morphology stability and chemical-physical properties of the metal nanoparticles are strongly influenced by the experimental conditions, the kinetics of the interaction of metal ions with reducing agents and the adsorption processes of stabilizing agents with metal nanoparticles. Hence, the design of the synthesis method in which the size morphology, stability and properties are controlled has become a major field of interest $\mathrm{t}^{3-5}$. There are many established wet-chemical methods for the synthesis of metal nanoparticles. Most of these methods are quite easy to execute and offer facile means to control the shape, sizes and surface characteristics of the produced nanoparticles, enabling scientists to precisely tune the overall physico-chemical properties of nanoparticles However, inspite of these advances, these techniques still have major drawbacks, e.g., use of starting materials, stabilizers and solvents used which are toxic and potentially hazardous. Moreover, adsorption of the toxic by-products on the surface of the synthesized nanoparticles is another problem associated with wet chemical methods $^{6-9}$.

Metal nanoparticles are currently being used in biomedical applications, such as novel biosensing devices and cancer therapy. For these applications, synthesized nanoparticles need to be biocompatible, water soluble and easily functionalized for fine-tuning their surface chemistry. However, biocompatibility can be severely compromised if toxic by-products formed during nanoparticle synthesis are adsorbed onto the particle surface. Biological molecules have qualities by which they can undergo highly controlled and hierarchical assembly, which makes them suitable for the development of a reliable and eco-friendly process for metal nanoparticles synthesis ${ }^{10,11}$. Therefore, the biological approach for the synthesis of nanoparticles becomes essential. One of the promising alternative synthetic routes for metal nanoparticles is biogenic synthesis, which employs non-toxic reactants derived from the biological 
sources ranging from unicellular organisms to higher plants. The key advantage of the use of plant extracts as the biogenic agents for metal nanoparticles synthesis is their easy availability. Moreover, simple laboratory set-up is required for the synthesis process and use of these biogenic materials potentially eliminates the elaborate process of cell culture and cell maintenance necessary for the biogenic synthesis of metal nanoparticles using unicellular organisms. Moreover, this synthetic protocol is applicable at room temperature and pressure, thus saving huge amount of energy.

Silver nanoparticles have unique properties that find its way in different applications viz.: (a)in assay as biological tags and biosensors in diagnostic applications, (b) incorporating AgNPs in apparel, footwear, wound dressings, paints, cosmetics and plastics resulted in antibacterial properties and (c) AgNPs are used in conductive inks and when incorporated in composites enhanced thermal and electrical ${ }^{12,13}$. Applications of the significant features of silver have increased since ancient times and due to the conveniently distributed sizes and shapes as well as a large surface area, silver nanoparticles are known as desirable and effective substances in many fields. AgNPs have received attention due to the surface plasmon resonance (strong absorption in the visible region), which can be easily monitored by UV-visible spectrophotometer. The applications of AgNPs in the field of medicine, optoelectronics, optics, catalysis, sensors are well known ${ }^{14-17}$.

Bio-inspired approaches were explored in the synthesis and applications of AgNPs using plant leaf extracts such as Crossandra infundibuliformi ${ }^{18}$, Acalypha indica ${ }^{19}$, Rhizophora mucronata $^{20}$, Mentha piperita ${ }^{21}$, Azadirachta indica ${ }^{9}$, lemongrass plant extract ${ }^{22}$, Stevia rebaudiana ${ }^{23}$, Chenopodium album $^{24}$, Cassia fistula ${ }^{25}$, Banana peel extract ${ }^{26}$, Alternanthera sessilis (Linn.) ${ }^{27}$ and Ocimum sanctum ${ }^{28}$. These AgNPs produced show good antimicrobial activity against common pathogens. Many reports have been published in the literature on the biogenesis of AgNPs using several plant seed extracts, particularly Papaver somniferum ${ }^{29}$, Jatropha curcas $^{30}$ and Medicago sativa ${ }^{31}$. In this context, we have designed a facile, biosynthetic route for the production of silver nanoparticles employing a biodegradable aqueous extract of natural plant seed as both the reducing and stabilizing agent. The present study involved the synthesis, characterization, in vitro antioxidant and antibacterial studies of silver nanoparticles. We have also evaluated the antibacterial activity of the gold nanoparticles from same plant source and results were well encouraged $^{32}$. So far, till date there is no report for silver nanoparticles and their antioxidant studies from the aqueous extract of Senna siamea plant seed. However prepared silver nanoparticles exhibited well toxicity against to gram-positive and gram-negative bacteria than gold nanoparticles.

\section{EXPERIMENTAL}

Preparation of aqueous extract of plant seed: Fresh $50 \mathrm{~g}$ seeds of Senna siamea plants were collected and washed with double distilled water. Cut into small pieces, transferred to $500 \mathrm{~mL}$ beaker and boiled with $300 \mathrm{~mL}$ of distilled water for $10 \mathrm{~min}$ up to $70{ }^{\circ} \mathrm{C}$ using magnetic stirrer. The obtained mixture was cooled and filtered through Whatman No. 1 filter paper. The boiled extract was refrigerated and used for further experimental procedures.

Biosynthesis of silver nanoparticles: $1 \mathrm{mM}\left(1 \times 10^{-3}\right)$ solution of silver nitrate $\left(\mathrm{AgNO}_{3}\right)$ stock solution was prepared by using sterile deionized triple distilled water. A series of volume $(2-10 \mathrm{~mL})$ of plant seed extract was added to $5 \mathrm{~mL}$ of $1 \times 10^{-3} \mathrm{M}$ aqueous $\mathrm{AgNO}_{3}$ solution and water being added to acquire a volume of $15 \mathrm{~mL}$. After $2 \mathrm{~h}$ incubation period at room temperature conditions, colour changed from transparent light yellow to brown colour indicating the formation of silver nanoparticles. The occurrence of colour was a primary indication of the formation of silver nanoparticles.

Ultraviolet-visible spectroscopy analysis: UV-Visible spectroscopy measurement was carried out on JASCO V 550 spectrophotometer operated at a resolution of $1 \mathrm{~nm}$.

FT-IR analysis: Identification of possible biomolecules responsible for the reduction and stabilization of silver nanoparticles was recorded using ABB MB3000 FT-IR spectrophotometer in the range of $4000-400 \mathrm{~cm}^{-1}$.

X-Ray diffraction studies: The phyto reduced silver colloidal solution was drop-coated onto a glass substrate and the XRD measurements were carried out using a Philips X'Pert Pro X-ray diffractometer, with the following working conditions: $\mathrm{CuK}_{\alpha}$ Ni-filtered radiation; $40 \mathrm{kV}, 30 \mathrm{~mA}$; divergence slit $0.47^{\circ}$

High resolution transmission electron microscopy (HRTEM) analysis: The stable biogenic AgNPs were washed and diluted by distilled water to attain the absorbance range of 0.5. Further, one drop of diluted AgNPs sample was placed on $\mathrm{Cu}$ grid with Ultrathin $\mathrm{Cu}$ on holey carbon disc and was allowed to dry in vacuo. After drying, the nanoparticles were visualized using JEOL JEM 2100 high resolution transmission electron microscope operating in the range of $200 \mathrm{kV}$ of acceleration.

Dynamic light scattering (DLS) and zeta potential measurements: Particle size measurement and zeta potential experiments were carried out by means of laser diffractometry, using zeta sizer nano-series (Malvern). Dynamic light scattering (DLS) was used for measurement of average hydrodynamic diameters and poly dispersity indexes (PDIs) (Malvern Zetasizer Nano-ZS, Malvern Instruments, UK). Each sample was analyzed in triplicate at $20^{\circ} \mathrm{C}$ at a scattering angle of $173^{\circ}$. Pure water was used as a references dispersing medium. The instrument was calibrated with Malvern-50V standard before each analysis cycle.

2,2-Diphenyl-1-picrylhydrazyl (DPPH) free radical scavenging assay: In vitro antioxidant activity was studied by testing the scavenging capacities on 2,2-diphenyl-1picrylhydrazyl (DPPH) free radical. The nanoparticle solution was lyophilized and makes it as a powder, the concentration of the stock solution $1 \mathrm{mg} / \mathrm{mL}$ concentration. Various concentrations of the stock solutions (concentrations of 10-50 $\mu \mathrm{L}$ ) were mixed with $0.25 \mathrm{mM}$ DPPH in ethanol, to produce a final DPPH concentration of $0.1 \mathrm{mM}$. The mixture was vigorously shaken and left to stand for $20 \mathrm{~min}$ in the dark and its absorbance was measured at $517 \mathrm{~nm}$. Quercetin was used as the standard. The radical-scavenging activities of samples, expressed as percentage inhibition of DPPH, were calculated according to the formula: 
Inhibition percentage $=$

$$
=\frac{\text { Absorbance of control }- \text { Absorbance of sample }}{\text { Absorbance of control }} \times 100
$$

Antibacterial activity: Antibacterial activity of the AgNPs was screened against two gram positive (Staphyloccus aureus and Bacillus subtilis) and three gram negative (Escherishea coli, Pseudomonas aeruginosa and Klebsiella pneumonia) human pathogenic bacteria using the agar well diffusion assay method. Gentamycin used as a standard for this antibacterial assay. About 18-20 mL of molten and cooled nutrient agar media was poured into sterilized petri dishes. The plates were left overnight at room temperature to allow any contamination to appear. The bacterial test organisms were grown in nutrient broth for 24 h. A $100 \mathrm{~mL}$ nutrient broth culture of each bacterial organism was used to prepare bacterial lawns. Agar wells with diameters of $5 \mathrm{mM}$ were prepared with the help of a sterilised stainless steel cork borer. The plates containing the bacterial and AgNPs were incubated at $37^{\circ} \mathrm{C}$ and then examined for evidence of zones of inhibition, which appear as a clear area around the wells. The diameter of such zones of inhibition was measured for each organism was recorded and expressed in millimetres.

\section{RESULTS AND DISCUSSION}

The formation of nanoparticles was easily detected and characterized by UV-visible spectroscopy owing to the surface plasmon resonance (SPR), i.e., the interaction of electromagnetic radiation and the electrons in the conduction band around the nanoparticles ${ }^{33}$. Under the UV region, AgNPs give a characteristic absorbance band due to the excitation mode of their surface plasmons which is dependent on the nanoparticle size. The spectrum can exhibit a shift towards the red end or the blue end depending upon the particle size shape, state of aggregation and the surrounding dielectric medium ${ }^{34-36}$. According to Henglein, the surface plasmon resonance band shifted to the blue when electrons are donated to the particles and the surface plasmon resonance band shifted to the red when holes are injected to the clusters ${ }^{37}$. The present study demonstrates the mechanism involved in the reduction of $\mathrm{AgNO}_{3}$ to $\mathrm{AgNPs}$ by the phytochemical constituents of aqueous extract of Senna siamea plant seed. In the present work, the AgNPs are rapidly formed after $2 \mathrm{~h}$ incubation period by the addition of aqueous extract of Senna siamea seed, evident from the appearance of brownish yellow colour and the $\lambda_{\max }$ appeared at $440 \mathrm{~nm}$ is depicted in Fig. 1. Earlier reports stated that maximum absorbance occurred at $435 \mathrm{~nm}$ due to presence of silver particle ${ }^{38}$.

FTIR measurement of the nanoparticles was carried out to study the interaction with the biomolecules present in the extracts responsible for stabilization of the nanoparticles. The peak at 3232, 2920-2849, 1736, 1649-1545, 1457-1117 and $980-767 \mathrm{~cm}^{-1}$ corresponds to polyphenols, aromatic C-H stretching, $\mathrm{N}-\mathrm{H}$ bending, $\mathrm{C}=\mathrm{N}$ stretching of aliphatic amines, aromatic $\mathrm{C}-\mathrm{H}$ bending of seed extracts Fig. 2(a) FT-IR spectrum suggests that the protein present in extract may be acts as capping agent and reducing agent. FT-IR spectrum of synthesized AgNPs were shown in Fig. 2(b). The absorption bands that appear AgNPs stabilized action with $\mathrm{N}-\mathrm{H}$ and $\mathrm{C}=\mathrm{N}$

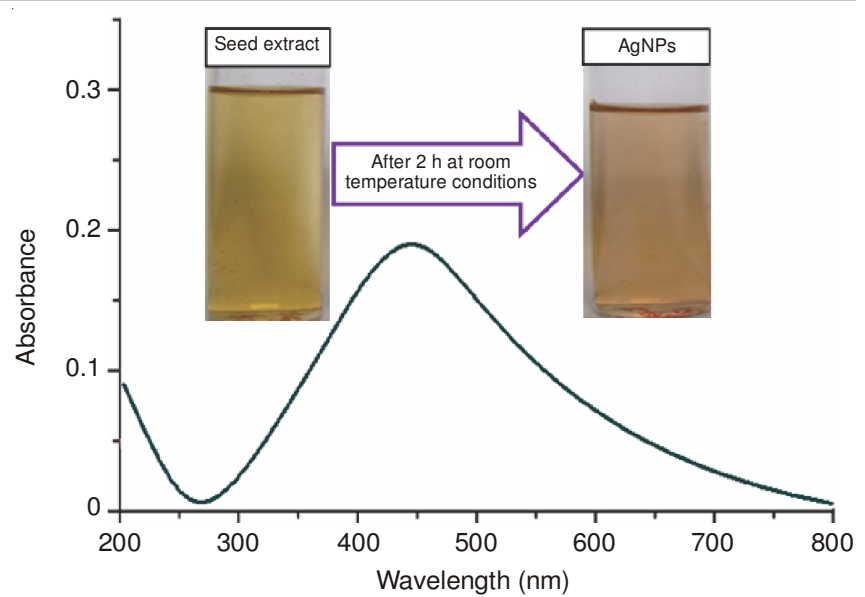

Fig. 1. UV-visible spectral (surface plasmon resonance band) analysis of bio synthesized silver nanoparticles

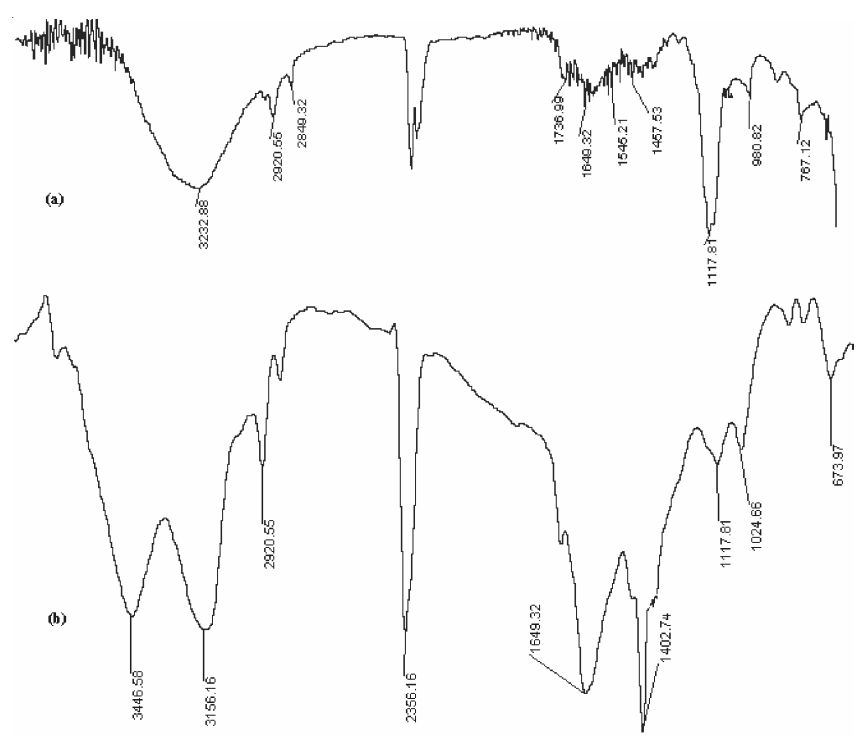

Fig. 2. (a) FT-IR spectrum of Senna saimea plant seed aqueous extract (b) FT-IR spectrum of synthesized silver nanoparticles

stretching of amines which is confirmed by FT-IR spectrum. IR spectrum peaks $\left(1649,1402\right.$ and $\left.1117 \mathrm{~cm}^{-1}\right)$ of the seed aqueous extract could also be seen in the IR spectra of phytocapped AgNPs. Furthermore the result shows that the phytochemical constituents, particularly protein and amines groups protect the AgNPs from aggregation and thereby retain the long term stability of nanoparticles ${ }^{38}$.

The crystalline nature of silver nanoparticles was confirmed from X-ray diffraction analysis. The typical powder XRD patterns of the prepared nanoparticles, originating from biosynthesis of different plant extracts, showed diffraction peaks at $2 \theta=38.2,44.4,64.6,77.5$ and $81.7^{\circ}$, which could be indexed to (1 11 1), (2 00 ), (2 20 ), (3 111 ) and (222) planes of pure silver (PDF No. 04-0783). This is confirmed that the main composition of the nanoparticles were of silver with fcc (face central cubic) lattice structure. The XRD pattern of the dried silver nanoparticles synthesized using Senna siamea plant seed aqueous extract is shown in Fig. 3. The three broad diffraction peaks were observed and indexed to the (111), (200) and (220) reflection planes of face centered cubic (fcc) structure of metallic silver nanopowers. All these peaks were well matched 


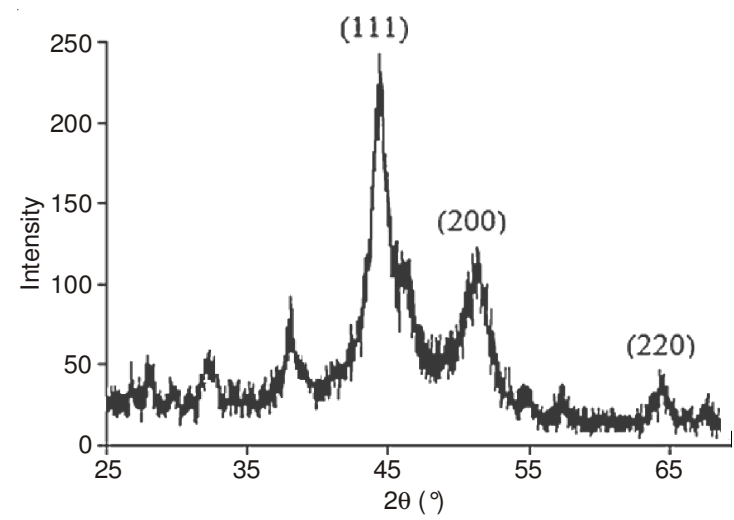

Fig. 3. XRD pattern of biosynthesized AgNPs exhibiting the facets of crystalline silver

with the standard JCPDS file No. 04-0783. The recorded XRD pattern also showed some other additional unassigned peaks which might be due to the formation of the crystalline bioorganic compounds/ metalloproteins that could be present in the plant seed extracts and were corresponding to the standard JCPDS file No. 22-1560 and 22-1532. These sharp Bragg peaks might have resulted due to the reducing agent stabilizing the nanoparticles. These peaks are much weaker than those of silver, which indicates that the silver is the main material in the composite compared with those of the bulk counterpart. Crystalline nature of the nanoparticles is further evidenced by the selected-area electron diffraction (SAED) patterns with bright circular spots corresponding to (111), (200) and (220) Bragg reflection planes shown in Fig. 4(b). XRD pattern shows that as metal ion concentration decreases the peaks become broadened. Generally, the broadening of peaks in the XRD patterns of solids is attributed to particle size effects ${ }^{39}$. Broader peaks signify smaller particle size and reflect the effects due to experimental conditions on the nucleation and growth of the crystal nuclei ${ }^{40}$.

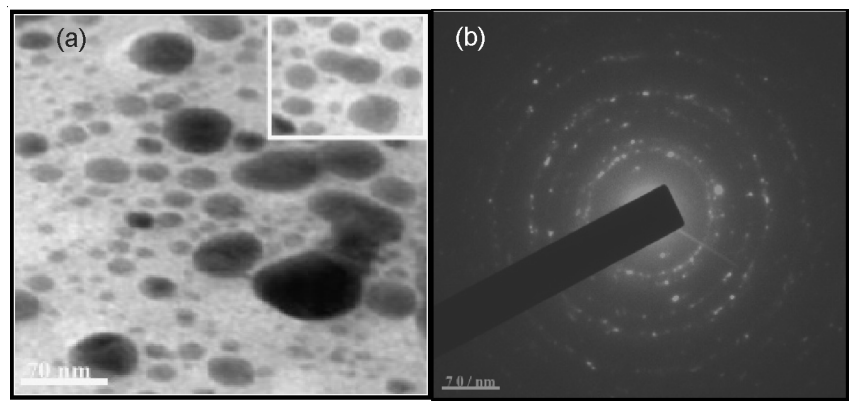

Fig. 4. (a) TEM image of silver nanoparticle (b) SAED pattern of silver nanoparticles

The application of transmission electron microscopy in nanosciences is significant to view the particles in nanoscale. The high resolution transmission electron microscopy (HRTEM) images of synthesized AgNPs and SAED pattern obtained from HRTEM studies are depicted in Fig. 4(a-b), which give clear indications regarding size, shape and size distribution of nanoparticles. From the images, it can be seen that the AgNPs are capped with phytoconstituents of Senna siamea seeds and TEM image is the evident that the morphology of silver nanoparticles is nearly spherical shape. The SAED pattern of AgNPs reveals its crystalline nature (white dots in Fig. 4). The size of the synthesized spherical shaped AgNPs were approximately $70 \mathrm{~nm}$ in size.

From the dynamic light scattering results, the calculated average particle size distribution of AgNPs is $70 \mathrm{~nm}$ and the corresponding average zeta potential value is $-22.9 \mathrm{mV}$ (Fig. 5(a-b)). A well dispersed AgNPs were found with respect to intensity. The results suggesting higher stability of AgNPs with out any changes in absorbance spectrum. The large negative potential value could be due to the capping of protein bio mass constituents present in the extract ${ }^{41}$.
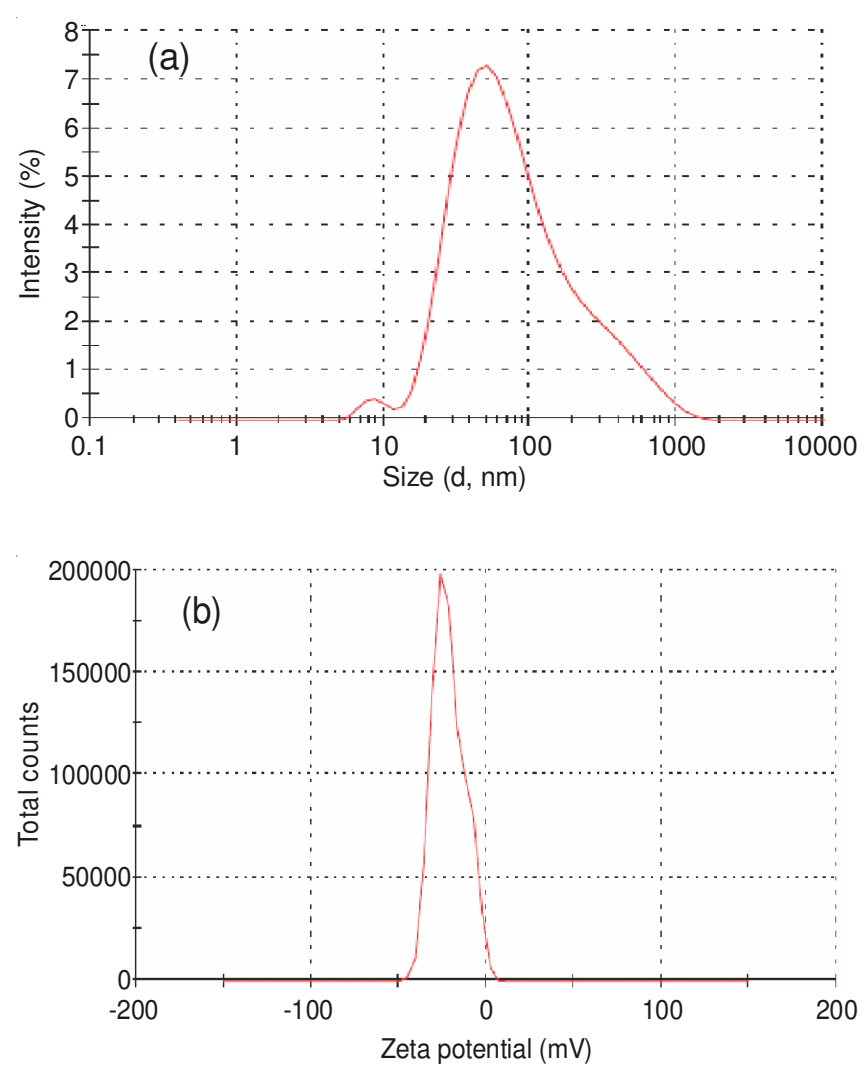

Fig. 5. (a) Size distribution by intensity of silver nanoparticles (b) Effective zeta potential of silver nanoparticles

For obtained protein capped silver nanoparticles, we evaluated in vitro antioxidant activity against to 2,2-diphenyl1-picrylhydrazyl (DPPH) free radical. The hydrogen atom or electron donation ability of the silver nanoparticles and the pure Senna siamea extract were measured from the bleaching of a purple-coloured ethanol solution of DPPH. Because DPPH and peroxyl radicals have similar electronic structures (the unpaired electron is delocalized over both $\mathrm{N}$ atoms of hydrazyl and both $\mathrm{O}$ atoms of peroxyl), the reaction rate of DPPH and antioxidants gives a good approximation for scavenging activities with lipid peroxyl radicals. This spectrophotometric assay uses the stable radical 2,2-diphenyl-1-picrylhydrazyl (DPPH) as a reagent. Antioxidant property was evaluated using DPPH which showed increase in free radical scavenging activity of the silver nanoparticles, which increased with the increase in concentration of the nanoparticles. Free radical scavenging activity of the silver nanoparticles was assessed 
by DPPH assay. The freshly prepared DPPH solution exhibited a deep purple colour with a maximum absorbance at $517 \mathrm{~nm}$. The disappearance of purple colour on adding synthesized silver nanoparticles might be due to presence of antioxidant in the medium. Free radical scavenging activity of the AgNPs on DPPH radical was found to increase with increase in concentration, showing a maximum of $62 \%$ at $500 \mu \mathrm{g} / \mathrm{mL}$. The standard quercetin, however, at this concentration exhibited $80 \%$ inhibition (Fig. 6).

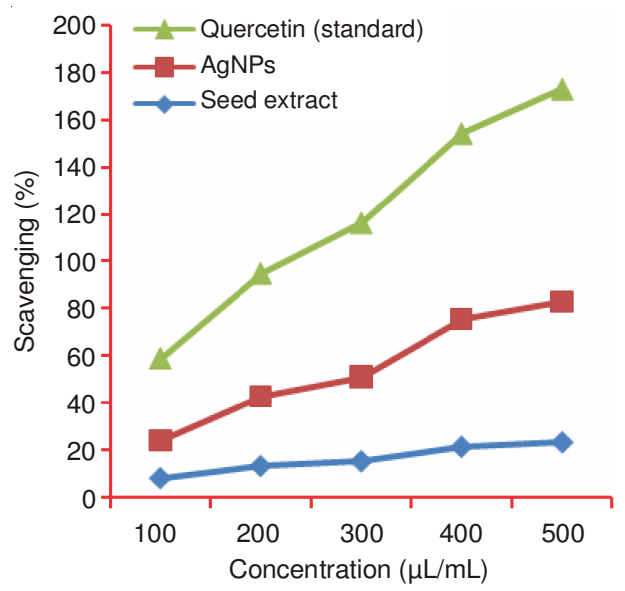

Fig. 6. DPPH free radical scavenging assay of protein capped silver nanoparticles

Biologically synthesized protein capped nanoparticles showed better antimicrobial activity against the growth of pathogen (Fig. 6). Aqueous extract of seed and silver nanoparticles were applied in 50,100 and $150 \mu \mathrm{L}$ concentrations in agar well diffusion method. The pathogens, Staphylococcus aureus $(20 \mathrm{~mm})$ and Pseudomonas aeruginosa $(18 \mathrm{~mm})$ found to be sensitive against the compound. And followed Bacillus subtilis (16 mm) Escherichia coli (15 mm), Klebsiella pneumoniae $(13 \mathrm{~mm})$ and shown in Fig. 7. For these silver nanoparticles Klebsiella pneumoniae shows noted resistance wich may be due to its strong cell wall chemistry. It Thus silver nanoparticles exhibit a broad size distribution and morphologies with highly reactive facets.

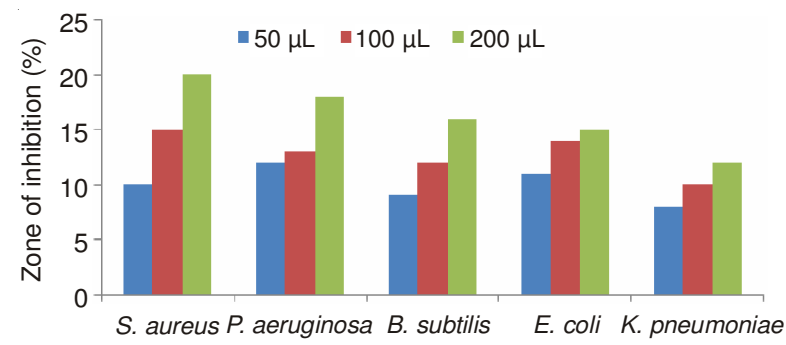

Fig. 7. Antibacterial activity of protein capped silver nanoparticle

It is well known that $\mathrm{Ag}^{+}$ions and $\mathrm{Ag}$-based compounds have strong antimicrobial effects and many investigators are interested in using other inorganic nanoparticles as antibacterial agents. These inorganic nanoparticles have a distinct advantage over conventional chemical antimicrobial agents. The most important problem caused by the chemical antimicrobial agents is multidrug resistance. Generally, the antimicrobial mechanism of chemical agents depends on the specific binding with the surface and the metabolism of agents into the microorganism. To date, antimicrobial agents based on chemicals have been effective for therapy. However, they have been limited to use in medical devices and prophylaxis in antimicrobial facilities. Therefore, an alternative way to overcome the drug resistance of various microorganisms is needed. $\mathrm{Ag}^{+}$ions and $\mathrm{Ag}$ salts have been used for decades as antimicrobial agents in various fields because of their growthinhibitory capacity against microorganisms. Many other researchers have tried to measure the activity of metal ions against microorganisms. Some studies have reported that the positive charge on the $\mathrm{Ag}^{+}$ion is crucial for its antimicrobial activity through the electrostatic attractions between the negatively charged cell membrane of microorganisms and the positively charged nanoparticles. In contrast, that the antimicrobial activity of AgNPs on Gram-negative bacteria was dependent on the concentration of the Ag nanoparticles used and was closely associated with the formation of pits in the cell wall of bacteria. Following this, Ag nanoparticles accumulate in the bacterial membrane and cause permeability, resulting in cell death. And smaller particles having the larger surface area available for interaction will give more bactericidal effect than the larger particles ${ }^{43-45}$.

\section{Conclusion}

This study summarizes the impact of Senna saimea plant seeds aqueous extract as a biological reducing and capping agent for synthesis of silver nanoparticles. The reduction of silver ion peak was measured at $440 \mathrm{~nm}$ by UV-visible analysis. Biomolecules that capped on silver nanoparticles was identified as protein content of seed aqueous extract through FTIR studies. Crystalline nature of the $70 \mathrm{~nm}$ spherical shaped nanoparticles is evident from bright spots in the SAED pattern, clear lattice fringes in the high-resolution TEM images and peaks in the XRD pattern. Effective antioxidant studies against to DPPH free radical was opened new vistas in this green chemistry quest which will directly help to manufacture a novel protein capped silver nanoantioxidant. Zeta potential studies will give strong evidence that these silver nanoparticles have high stability. Present investigation also suggests that the biosynthesized silver nanoparticles are exerting in vitro toxic effect on human bacterial pathogens. Looking into all these aspects, it is reasonable to infer that the biosynthesis of spherical shaped silver nanoparticles hopefully might reach this aim because they display novel properties. The biological methods of synthesizing nanoparticles have proved to be one of the best methods so far. This is due to its environmentfriendly nature and slower kinetics which offer better manipulation and control over crystal growth and stabilization.

\section{REFERENCES}

1. Y.S. Jae and S.K. Beom, Bioprocess Biosyst. Eng., 32, 79 (2009).

2. A. Singh, D. Jain, M.K. Upadhyay, N. Khandelwal and H.N. Verma, Dig, J. Nanomater. Biostruct., 5, 659 (2010).

3. B. Knoll and F. Keilmann, Nature, 399, 134 (2010).

4. S. Sengupta, D. Eavarone, I. Capila, G.L. Zhao, T. Watson and T. Kiziltepe, Nature, 436, 568 (2005).

5. B.J. Wiley, Y. Sun and Y. Xia, Acc. Chem. Res., 40, 1067 (2007).

6. G. Palui, S. Ray and A. Banerjee, J. Mater. Chem., 19, 3457 (2009).

7. Z.C. Xu, C.M. Shen, C.W. Xiao, T.Z. Yang, H.R. Zhang and J.Q. Li, Nanotechnology, 18, 115608 (2007). 
8. N. Roay, S. Mondal, R.A. Laskar, S. Basu, D. Mandal and N.A. Begum, Colloids Surf. B, 76, 317 (2010).

9. S.S. Shankar, A. Rai, A. Ahmad and M. Sastry, J. Colloid Interf. Sci., 275, 496 (2004).

10. N.T. Kaushik, S.M. Snehit and Y.P. Rasesh, Nanomed. Nanotechnol. Biol. Med., 6, 257 (2010).

11. U.K. Parashar, P.S. Saxena and A. Srivastava, Dig. J. Nanomater. Biostruct., 4, 159 (2009).

12. W.R. Li, X.B. Xie, Q.S. Shi, H.Y. Zeng, Y.S. Ou-Yang and Y.B. Chen, Appl. Microbiol. Biotechnol., 85, 1115 (2010).

13. R. Dastjerdi, M. Montazer and S. Shahsavan, Colloids Surf. A, 345, 202 (2009).

14. J. Nam, N. Won, H. Jin, H. Chung and S. Kim, J. Am. Chem. Soc., 131, 13639 (2009).

15. K.B. Narayanan and N. Sakthivel, J. Hazard. Mater., 189, 519 (2011).

16. J. Li, X. Chen, N. Ai, J. Hao, Q. Chen and S. Strauf, Chem. Phys. Lett., 514, 141 (2011).

17. A.M. Fayaza, M. Girilal, S.A. Mahdy, S.S. Somsundar, R. Venkatesan and P.T. Kalaichelvan, Process Biochem., 46, 636 (2011).

18. S. Kaviya, J. Santhanalakshmi and B. Viswanathan, Mater. Lett., 67, 64 (2012).

19. C. Krishnaraj, E.G. Jagan, S. Rajasekar, P. Selvakumar, P.T. Kalaichelvan and N. Mohan, Colloids Surf. B, 76, 50 (2010).

20. M. Gnanadesigan, M. Anand, S. Ravikumar, M. Maruthupandy, V. Vijayakumar, S. Selvam, M. Dhineshkumar and A.K. Kumaraguru, Asian Pacif. J. Trop. Med., 4, 799 (2011).

21. D. MubarakAli, N. Thajuddin, K. Jeganathan and M. Gunasekaran, Colloids Surf. B, 85, 360 (2011).

22. M. Rai, A. Yadava and A. Gadea, Biotechnol. Adv., 27, 76 (2009).

23. M. Yilmaz, H. Turkdemir, MA. Kilic, E. Bayram, A. Cicek, A. Mete and B. Ulug, Mater. Chem. Phys., 130, 1195 (2011).

24. A.D. Dwivedi and K. Gopal, Colloids Surf. A, 369, 27 (2010).

25. L.Q. Lin, W.T. Wang, J.L. Huang, Q.B. Li, D.H. Sun, X. Yang, H.X. Wang, N. He and Y.P. Wang, Chem. Eng. J., 162, 852 (2010).
26. A. Bankar, B. Joshi, A.R. Kumar and S. Zinjarde, Colloids Surf. A, 368, 58 (2010).

27. K.L. Niraimathi, V. Sudha, R. Lavanya and P. Brindha Colloids Surf. B, 102, 288 (2013).

28. Y.S. Rao, V.S. Kotakadi, T.N.V.K.V. Prasad, A.V. Reddy and D.V.R. Sai Gopal, Spectrochim. Acta A, 103, 156 (2013).

29. K. Vijayaraghavan, S.P. Nalini, N.U. Prakash and D. Madhankumar, Colloids Surf. B, 94, 114 (2012).

30. H. Bar, D.K. Bhui, G.P. Sahoo, P. Sarkar, S. Pyne and A. Misra, Colloids Surf. A, 348, 212 (2009).

31. A.I. Lukman, B. Gong, C.E. Marjo, U. Roessner and A.T. Harris, J. Colloid Interf. Sci., 353, 433 (2011).

32. G.R. Reddy, A.B. Morais and N.N. Gandhi, Asian J. Chem., 25, 8541 (2013).

33. J. Park and Y. Kim, J. Nanosci. Nanotechnol., 8, 1 (2008).

34. D.J. Patel and V. Kumar, J. Pharm. Res., 1, 34 (2008).

35 A. Shirwaikar, K. Rajendran, C. Dinesh Kumar and R. Bodla, J. Ethnopharmcol., 91, 171 (2004).

36. E. Bulut, Ind. Eng. Chem. Res., 48, 5686 (2009).

37. A. Henglein, J. Phys. Chem., 97, 5457 (1993).

38. M. Sathishkumar, K. Sneha, S.W. Won, C. W. Cho, S. Kim and Y.S. Yun, Colloids Surf. B, 73, 332 (2009).

39. H. Borchert, E.V. Shevchenko, A. Robert, I. Mekis, A. Kornowski and G. Grubel, Langmuir, 5, 1931 (2005).

40. R. Jenkins and R.L. Snyder, Introduction to X-Ray Powder Diffractiometry, John Wiley \& Sons, New York, p. 544 (1996).

41. A. Becheri, M. Durr, P.L. Nostro and P. Baglioni, J. Nanopart. Res., 10, 679 (2008).

42. P. Dibrov, J. Dzioba, K. K. Gosink and C. C. Häse, Antimicrobial. Agents Chemother, 46, 2668 (2002).

43. I. Dragieva, S. Stoeva, P. Stoimenov, E. Pavlikianov and K. Klabunde, Nanostructured Mater., 12, 267 (1999).

44. C. Baker, A. Pradhan, L. Pakstis, J. Pochan Darrin and S.S. Ismat, J. Nanosci. Nanotechnol., 5, 244 (2005). 